\title{
Safety and efficacy of conservative, endovascular bare stent and endovascular coil assisting bare stent treatments for patients diagnosed with spontaneous isolated superior mesenteric artery dissection
}

\author{
Abdala Maulid Mkangala, Huimin Liang, Xiang-Jun Dong, Yangbo Su, Lu HaoHao \\ Department of Radiology, Union Hospital, Tongji Medical College of Huazhong University of Science and Technology, Wuhan, \\ Hubei, China
}

Videosurgery Miniinv 2020; 15 (4): 608-619 DOI: https://doi.org/10.5114/wiitm.2020.92403

\begin{abstract}
Introduction: Isolated dissection of visceral artery organs is very infrequently reported and when it occurs it mostly affects the superior mesenteric artery (SMA) with abdominal pain as the commonest presenting symptom. However, the best therapeutic strategy in symptomatic patients has not yet been established.

Aim: To evaluate the safety and efficacy of conservative, endovascular bare stent and endovascular coil assisting bare stent treatments for patients diagnosed with spontaneous isolated superior mesenteric artery dissection (SISMAD).

Material and methods: We reviewed patients who had SISMAD and received conservative, bare stent and coil assisting bare stent as a primary treatment between 2014 and 2018. Patient demographics, symptoms, angiographic findings and treatment outcomes were analyzed.

Results: A total of 62 patients was found to have SISMAD among whom $83.9 \%(n=52)$ were male and $16 \%$ $(n=10)$ were female with the mean age of $52.55 \pm 7.22$ years, range $33-77.22 .6 \%(n=14)$ received conservative, $62.9 \%(n=39)$ endovascular bare stent and 14. Four percent $(n=9)$ endovascular coil assisting bare stent treatment. The success rate in primary treatment was conservative $78.5 \%(n=11)$, bare stent $97.4 \%(n=38)$, coil assisting bare stent $100 \%(n=9)$. The mean follow-up duration (months) was $28.76 \pm 12.87$.

Conclusions: Endovascular bare stent placement is a safe, effective, and successful treatment in the management of symptomatic SISMAD. The diagnostic imaging result is a key point for planning appropriate treatment especially in patients with tapered vessels, longer dissection lesion, and dissection aneurysm where coil assisting bare stent shows good results. Conservative treatment should be given priority for the asymptomatic patient, but close monitoring is highly recommended.
\end{abstract}

Key words: superior mesenteric artery dissection, endovascular treatment, bare stent, coil embolization, dissection aneurysm, abdominal pain.

\section{Introduction}

Spontaneous dissection that is confined to the superior mesenteric artery was considered to be an uncommon vascular disease with a fatal outcome
[1]. However, in recent years more patients have been detected following improvement in diagnostic radiological practices such as the development of multidetector computed tomography (MDCT), proceeding into multiplanar reconstruction (MPR) along 
with reformation imaging and computed tomography angiography (CTA) [1-5]. Currently, the preferred diagnostic radiological imaging in patients with subtle abdominal pain in the casualty is enhanced computed tomography (CT). CT is essentially very useful for the initial identification of the lesion and also in the course of follow-up because it minimizes the partial volume artifacts and reduced the misdiagnosis of the artery [5]. This is the result of the lengthwise orientation of superior mesenteric artery (SMA) located vertically to the studying plan $[6,7]$. From a broader perspective, CTA is more accurate, non-invasive and able to show clearly the arterial dissection, length of dissection, the extent, the scope and the involvement of the lumen with the ability to reveal the true and false lumen or presence of thrombosis and lumen stenosis.

Etiologically, atherosclerosis, medial cystic necrosis, fibromuscular dysplasia, as well as untreated hypertension, are reported to be risk factors [7]. Jiang et al. [8] reported 2 patients, an uncle and his nephew with Chinese ethnicity, both with superior mesenteric artery dissection (SMAD). Genetic analysis showed the linkage of these three family members to $5 q 13-14$, which was also found to be related to familial dissection and aneurysm of the ascending aorta. The common clinical presentation is abdominal pain, in particular acute or chronic epigastric pain, which is suggested due to the length of the lesion itself, intestinal ischemia or infarction with peritonitis [9]. Other presenting features are vomiting, nausea, diarrhea, and abdominal wall distention.

The main treatment goals are symptoms relief, restoration of blood circulation from proximal to distal via the true lumen, and prevention of false lumen extension and rupture. Different treatment approaches have been proposed such as conservative, endovascular and open surgical treatment. Conservative treatment mainly targets patients with no sign of bowel ischemia while the symptomatic patient's endovascular stenting and surgical repair have been recommended [10-12]. Mizuno et al. [13] demonstrated that conservative treatment of spontaneous isolated superior mesenteric artery dissection (SISMAD) patients presenting without aneurysm is safe and has a low rate of further surgical treatment so as the reports from the findings by Kwon et al. [14].

Currently, a significant number of articles describe endovascular stent treatment as a first treatment choice in symptomatic patients with good clinical outcomes [15, 16]. However, the debate still continues regarding the best choice between self-expandable bare or covered stent. Wen et al. [17] in their report presented 12 patients who successfully received a covered stent with good short term results. However, Chang et al. [18] suggested that based on branches formed along the SMA it is unavoidable to develop mesenteric ischemia in the long term period. A bare stent is flexible and has less radial force; therefore, it is suitable for the weak vascular wall and original curved site of the SMA. However, the previous report shows a high rate of thrombosis due to its ineffectiveness on the false lumen [19]. Furthermore, there is the issue of whether to apply a coil in the false lumen or not in the case of dissection aneurysm. Recently, in their report, Jia et al. [20] showed that using bare stents only and a stent with false lumen coil embolization both have high technical success rates and revealed good midterm patency in patients with dissection aneurysms. To date, the best endovascular therapeutic strategy in symptomatic patients not yet been concluded.

\section{Aim}

Our aim is to evaluate the safety and efficacy of conservative, endovascular bare stent and endovascular coil assisting bare stent treatments for the patients diagnosed with SISMAD.

\section{Material and methods}

\section{Patients' demographic and clinical presentation}

Our clinical research using retrospective medical record case review was allowed by the institutional review board, and hence the requirement for patient informed consent was waived. The hospital information electronic databases from diagnostic tests and notes from health care providers were reviewed for all patients who had SISMAD diagnoses according to enhanced CT and CTA imaging performed between 2014 and 2018. Patient demographics, clinical manifestation, associated risk factors, diagnostic imaging, treatment modalities, and outcomes were extracted by using a prepared review data table. All the information and imaging of a patient with SISMAD presented as symptomatic or asymptomatic who received treatment in our hospital with available follow-up imaging results were extracted. Our inclusion 
criteria were all patients with isolated lesion who underwent conservative treatment, endovascular bare stent placement or endovascular coil assisting bare stent. Patients who had a lesion extending to the aorta were excluded.

\section{Diagnosis}

In our study, the initial diagnosis was reached through contrast-enhanced CT (CECT) (Siemens definition AS 128 CT) (CTA) and digital subtraction angiography (DSA). Contrast-enhanced CT scanning was performed with a thickness section of $1.5 \mathrm{~mm}$
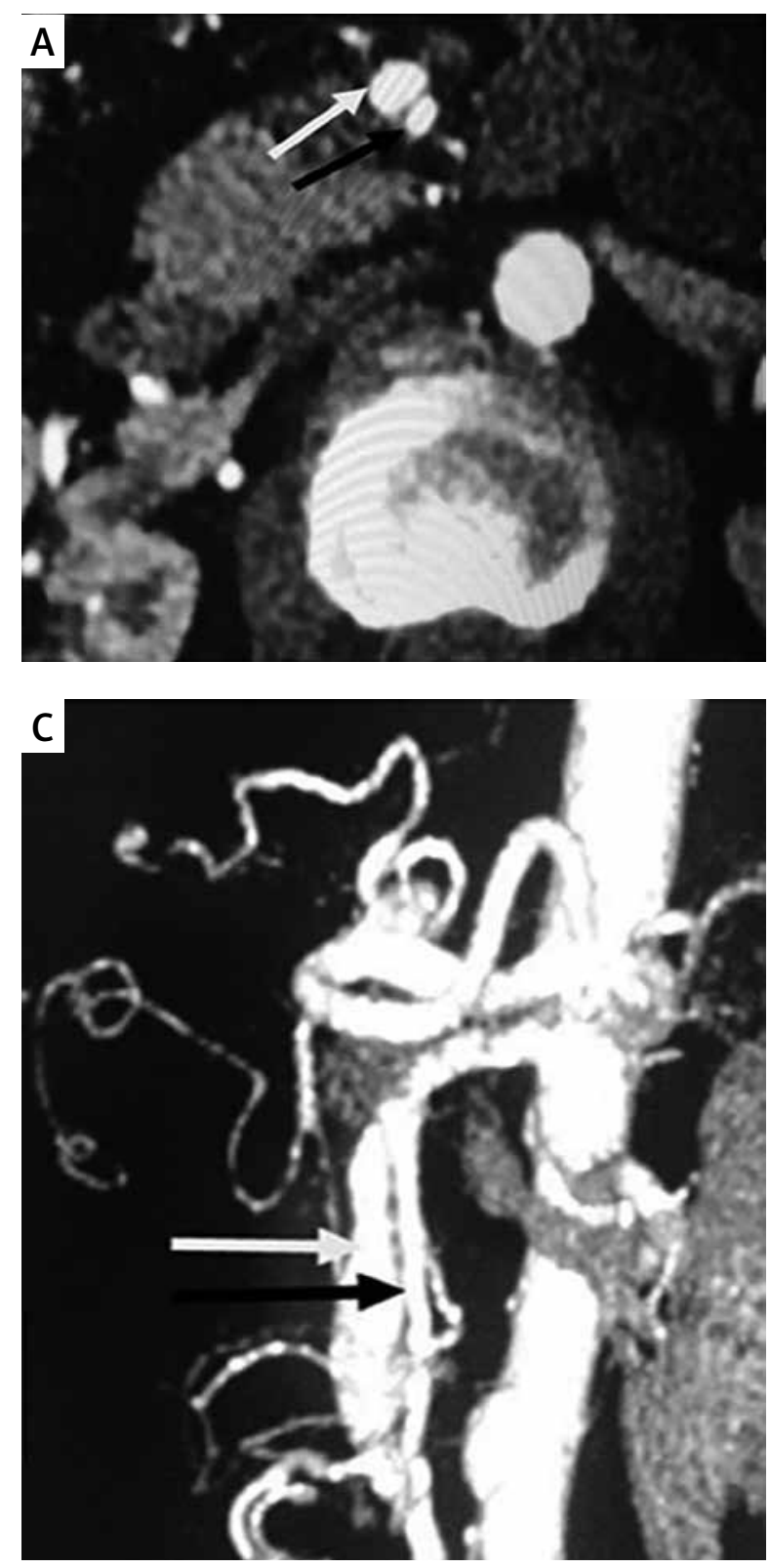

and a pitch of 1 and CTA section scanning of $1.5 \mathrm{~mm}$ and a pitch of 1 . The constructive data thickness section was $0.75 \mathrm{~mm}$ with an increment of $0.5 \mathrm{~mm}$. Post-processing image methods used on the working station were 3D volume rendering, multiplanar reconstruction, curved planar reconstruction and maximum intensity projection (MIP). Contrast-enhanced CT was performed to establish the diagnosis of SISMAD. The pathognomonic finding of SISMAD is the intimal flap (Photo $1 \mathrm{~A}$ ) and all cross-sectional modalities allow its identification. CT angiography was performed to establish the point of entry site,

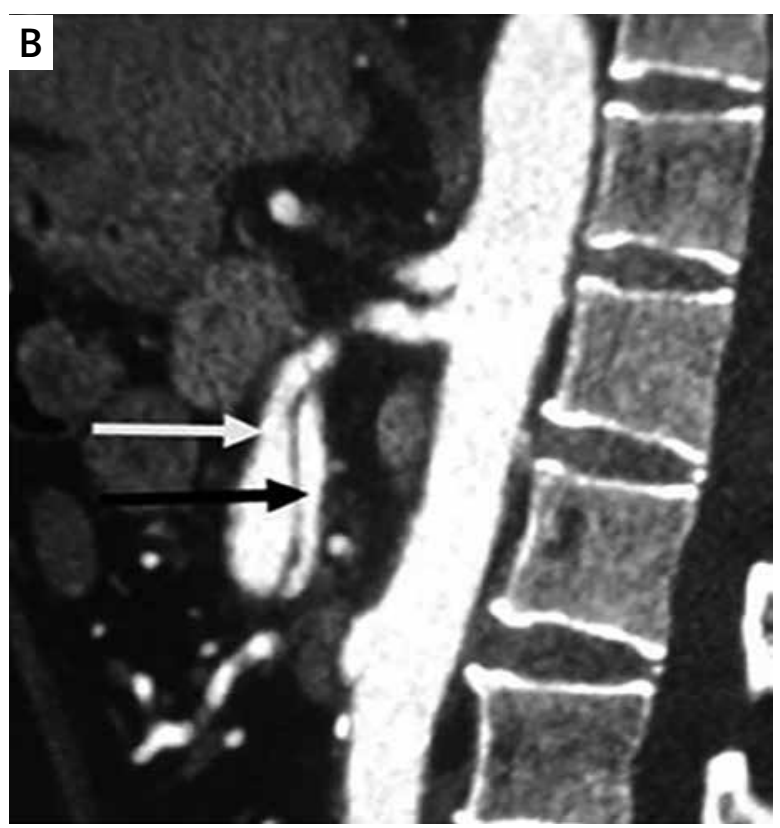

Photo 1. Diagnostic abdominal CTA: A-cross-section plane shows spontaneous isolated SMA dissection with true lumen (black arrow) separated by intimal flapping (the pathognomonic finding of SISMAD) from false lumens (white arrow), B - CT reconstruction, longitudinal view revealing the entry site, dissection length and presence of pseudo-aneurysm, C - VR image lateral views revealing SMA vascular branches 
lesion length, and presence of a false lumen or dissection/pseudo-aneurysm (Photos $1 \mathrm{~B}, \mathrm{C}$ ).

On angiographic images (Siemens Artis Zee Celling and Siemens Artis Zee Floor, Siemens Medical Solutions, Muenchen, Germany) and Philips Allura Xper FD20 Philips Medical System, Best, The Netherlands) the SISMAD was proved by seeing the filling of the contrast medium to the dissection sac with the same attenuation as its parent artery in the arterial phase. The presence of filling defects between the parent artery and the false lumen indicates the thrombus of the false lumen sac. The proximal and distal arteries to the dissection as well as its branches were confirmed by CTA and DSA images.

\section{Conservative treatment}

Conservative management was recommended for patients who presented as either asymptomatic or symptomatic with no sign of imminent arterial rupture or bowel ischemia. In our setup patients received either the non-medical intervention or medical intervention. Non-medical intervention consists of fasting, arterial blood pressure lowering in case it is higher, parenteral dietary support and close observation of patients for abdominal symptoms prognosis. In medical therapy, patients received antipain drugs to relieve pain and blood thinner drugs such as heparin (anticoagulants) and aspirin (antiplatelet). Heparin was administered in a dose of $1 \mathrm{mg} / \mathrm{kg}$ (two times a day) and aspirin $100 \mathrm{mg}$ (once a day). If a patient's prognosis was poor endovascular treatment was the second option.

\section{Endovascular treatment}

Endovascular procedures were performed by the team of experienced interventional radiologists un-
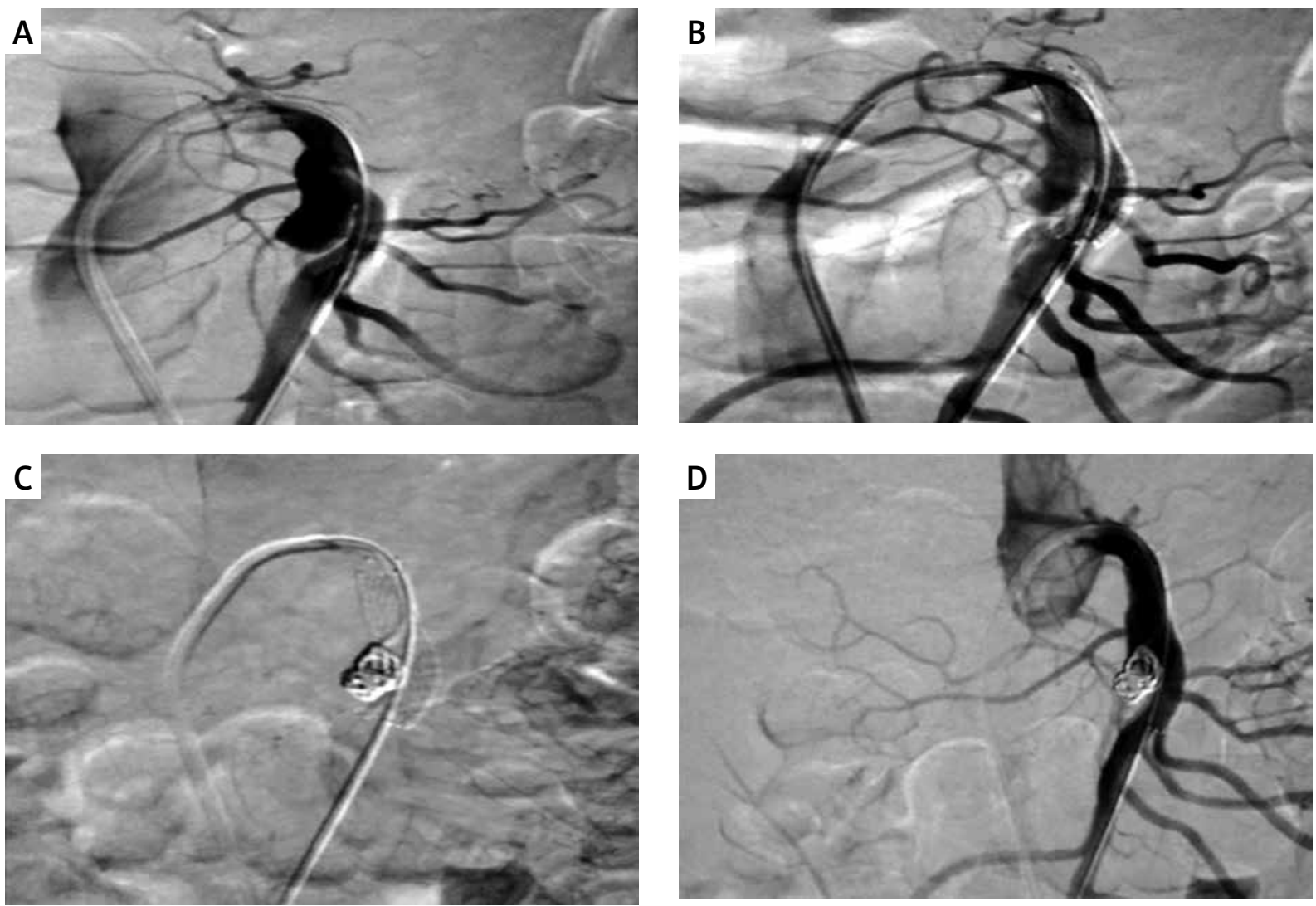

Photo 2. Endovascular coil assisting bare stent: $\mathbf{A}$ - angiography revealing the entry point of the true lumen and dissection aneurysm, $\mathbf{B}$ - angiography taken after bare stent deployment shows persistent dissection aneurysm, C - successful false lumen coil embolization, D - control angiography revealed good blood flow of the SMA from the proximal to the distal as well as side branches with successfully sealing of false lumen by the coil 
der the guidance of digital subtraction angiography through the modified Seldinger technique. The femoral artery was accessed by an $8 \mathrm{Fr}$ guiding catheter (Boston Scientific, Natick, Mass), to obtain a selective SMA arteriogram to reveal the entry and the length of dissection as well as the proximal and the distal flow of the side branches and dissection aneurysm morphology. Once it was confirmed that the lesion was not so close to the origin of the SMA and the false lumen was not occluding the true lumen the interventional radiologist guided a 0.035 " guidewire (Radifocus, Terumo Co., Tokyo, Japan) to cross the primary entry and access the true distal lumen, hence facilitating the pathway for stent deployment.

The bare self-expanding stents (Biotronik, Medtronic or BARD) were implanted over the opening site, typically from the distal to the proximal. If the dissection length was greater than the primary stent, the second stent was introduced in overlapping stent fashion. In cases of dissection aneurysm, coil assisting bare stent was the treatment of choice, whereby a microcatheter (Terumo) was guided to the neck of the false lumen and a coil (Cook Inc.) was introduced and packed in the false lumen cavity (Photos 2 A-D). In all cases, a 5000 IU bolus of heparin was administered before the procedure. Angiography study contrast media (Omnipaque 350; Ge Health Care, Shanghai, China) were used. The volumes of $25 \mathrm{ml}$ of contrast were injected at a flow rate of $5 \mathrm{ml} / \mathrm{s}$. The femoral access site was closed with Perclose ProGlide (Abbott, Chicago, III). Post-procedure aspirin (100 mg/day) and clopidogrel (75 mg/day) were prescribed for 3 months and then aspirin was continued depending on the patient responses.

\section{Follow-up}

After the primary intervention, outpatient clinic attendances were insisted where the complications, morbidity and mortality rates of treatment were recorded. Follow-up guidelines included history and clinical examination followed by CT at 1, 6 and 12 months and yearly thereafter. Successful endovascular management was defined as a primary and secondary outcome. Initial success was defined as normal blood supply to the distal SMA being restored, and symptoms being relieved. Secondary success is when the false lumen (pseudo-aneurysms) is obliterated with a patent stent or remodeling of false lumen on final follow-up CTA angiography. Patients were considered as lost cases during the follow-up process if they missed two follow-up radiological studies after final procedures.

\section{Statistical analysis}

In our study, statistical analyses were performed using the IBM SPSS Statistics 20 software. All of our continuous data were presented as mean \pm standard deviation (SD) and categorical data were presented as a percentage. The comparison was made between the groups by one-way ANOVA test for independent measures and the $\chi^{2}$ test method. A $p$-value of $<0.05$ was defined as statistically significant.

\section{Results}

The general demographic, clinical characteristics, diagnosis and treatment outcome of our 62 patients are summarized in Table I. Among 62 patients $83.9 \%$ $(n=52)$ were male and $16 \%(n=10)$ were female with the mean age of $52.55 \pm 7.22$ years, range: $33-77.72 .6 \%(n=45)$ were symptomatic and $27.4 \%$ $(n=17)$ were asymptomatic patients with mean duration of onset to admission $5.03 \pm 4.04$ days, range: 1-19. All symptomatic patients presented with abdominal pain $72.6 \%(n=45)$, nausea $51.6 \%(n=32)$, vomiting $30.6 \%(n=19)$ diarrhea $22.6 \%(n=14)$ and blood in stool $16.1 \%(n=10)$. Relevant associated co-morbidities included hypertension $41.9 \%(n=26$, atherosclerosis $41.9 \%(n=26)$, smoking $25.8(n=16)$. About $87 \%$ of our cases were diagnosed as SISMAD by enhanced CT and $13 \%$ were diagnosed by CTA. The mean length of SMA dissection was $4.72 \pm 1.96$ (range: 1.50-11.20). The mean distance from the SMA origin to the dissection entry point was 2.49 $\pm 1.06 \mathrm{~cm}$ (range: $0.60-5.60$ ). Based on angiographic findings our patients fall into: type I, $17.7 \%(n=11)$, type IIA, 58.1\% $(n=36)$ and type IIB, $24.2 \%(n=15)$ based on the modified Sakamoto classification.

Among 62 patients $22.6 \%(n=14)$ received conservative treatment, $62.9 \%(n=39)$ an endovascular bare stent and $14.4 \%(n=9)$ received an endovascular coil assisting a bare stent. Based on these three groups, the duration of onset to admission was higher in the conservative group compared to the endovascular bare stent group and endovascular coil assisting bare stent group; $p=0.001$ and $p=0.030$ respectively (Tables $I-I V)$. The length of dissection is longer in both the endovascular bare stent and endovascular coil assisting bare stent 
Table I. General demographic characteristics, symptoms, angiographic findings, treatment and outcome $(n=62)$

\begin{tabular}{|c|c|c|c|c|}
\hline Variables & Total $(n=62)$ & Conservative $(n=14)$ & Stenting $(n=39)$ & $\begin{array}{c}\text { Stenting + coiling } \\
(n=9)\end{array}$ \\
\hline Age & $52.55 \pm 7.22$ & $52.64 \pm 5.79$ & $51.84 \pm 8.10$ & $55.44 \pm 4.33$ \\
\hline \multicolumn{5}{|l|}{ Sex: } \\
\hline Male & $52(83.9)$ & $12(85.7)$ & $31(79.5)$ & $9(100)$ \\
\hline Female & $10(16.1)$ & $2(14.3)$ & $8(20.5)$ & 0 \\
\hline \multicolumn{5}{|l|}{ Symptoms: } \\
\hline Asymptomatic & $17(27.4)$ & $11(78.6)$ & $6(15.4)$ & $0(0)$ \\
\hline Symptomatic & $45(72.6)$ & $3(21.4)$ & $33(84.6)$ & $9(100)$ \\
\hline Duration of symptoms & $5.03 \pm 4.04$ & $8.81 \pm 5.90(1-19)$ & $3.97 \pm 2.59$ & $3.89 \pm 1.97$ \\
\hline Abdominal pain & $45(72.6)$ & $3(21.4)$ & $33(84.6$ & $9(100)$ \\
\hline Nausea & $32(51.6)$ & $2(14.3)$ & $25(64.1)$ & $5(33.3)$ \\
\hline Vomiting & $19(30.6)$ & $2(14.3)$ & $14(35.9)$ & $3(33.3)$ \\
\hline Diarrhea & $14(22.6)$ & $2(14.3)$ & $8(20.5)$ & $2(22.2)$ \\
\hline Blood in stool & $10(16.1)$ & 0 & $6(15.4)$ & $4(44.4)$ \\
\hline \multicolumn{5}{|l|}{ Risk factor: } \\
\hline Hypertension & $26(41.9)$ & $3(21.4)$ & $19(48.7)$ & $4(44.4)$ \\
\hline Atherosclerosis & $26(41.9)$ & $3(21.4)$ & $18(46.2)$ & $4(44.4)$ \\
\hline Smoking & $16(25.8)$ & $2(14.3)$ & $12(30.8)$ & $2(22.2)$ \\
\hline Diabetes mellitus & 0 & 0 & 0 & 0 \\
\hline \multicolumn{5}{|l|}{ Angiographic findings: } \\
\hline Type I & $11(17.7)$ & $7(50)$ & $4(10.3)$ & 0 \\
\hline Type IIA & $36(58.1)$ & $2(14.3)$ & $25(64.1)$ & $9(100)$ \\
\hline Type IIB & $15(24.2)$ & $5(35.7)$ & $10(25.6)$ & 0 \\
\hline Dissection length $[\mathrm{cm}]$ & $4.72 \pm 1.96$ & $3.60 \pm 1.49$ & $5.09 \pm 2.13$ & $4.86 \pm 1.08$ \\
\hline Distance from A-L [cm] & $2.49 \pm 1.06$ & $2.09 \pm 0.77$ & $2.68 \pm 1.19$ & $2.33 \pm 0.52$ \\
\hline \multicolumn{5}{|l|}{ Treatment: } \\
\hline Single stent & $41(66.1)$ & 0 & $34(87.2)$ & $7(77.8)$ \\
\hline Overlapping stent & $7(11.3)$ & 0 & $5(12.8)$ & $2(22.2)$ \\
\hline Diameter of stent & $7.06 \pm 0.56$ & 0 & $7.10 \pm 0.59(6-8)$ & $6.89 \pm 0.33(6 .-7)$ \\
\hline Length of stent & $64.38 \pm 23.69$ & 0 & $66.41 \pm 25.18$ & $55.56 \pm 13.33$ \\
\hline Fasting days & $7.52 \pm 6.61(3-28)$ & $17.07 \pm 8.51$ & $5.13 \pm 1.30$ & $4.11 \pm 0.60$ \\
\hline \multicolumn{5}{|l|}{ Outcome: } \\
\hline Success & $58(93.5)$ & $11(79)$ & $38(97.4)$ & $9(100)$ \\
\hline Failure & $4(6.5)$ & $3(21)$ & $1(2.6)$ & 0 \\
\hline Secondary treatment & $4(6.5)$ & $3(21)$ & $1(2.6)$ & 0 \\
\hline Follow-up & $28.76 \pm 12.87$ & $26.50 \pm 12.95$ & $31.46 \pm 13.15$ & $20.56 \pm 6.98$ \\
\hline
\end{tabular}

$A-L$ (distance from aorta to lesion entry point). 
Table II. Conservative treatment group vs. endovascular bare stent treatment group (demographic characteristics, symptoms, angiographic findings, treatment and outcome)

\begin{tabular}{|c|c|c|c|}
\hline Variables & Conservative $(n=14)$ & Stenting $(n=39)$ & $P$-value \\
\hline Age & $52.64 \pm 5.79$ & $51.84 \pm 8.10$ & 0.737 \\
\hline \multicolumn{4}{|l|}{ Sex: } \\
\hline Male & $12(85.7)$ & $31(79.5)$ & 0.609 \\
\hline Female & $2(14.3)$ & $8(20.5)$ & \\
\hline \multicolumn{4}{|l|}{ Clinical presentation: } \\
\hline Asymptomatic & $11(78.6)$ & $6(15.4)$ & $0.001^{\star}$ \\
\hline Symptomatic & $3(21.4)$ & $33(84.6)$ & \\
\hline Duration of symptoms & $8.81 \pm 5.90$ & $3.97 \pm 2.59$ & $0.001^{*}$ \\
\hline Abdominal pain & $3(21.4)$ & $33(84.6)$ & $0.001^{\star}$ \\
\hline Nausea & $2(14.3)$ & $25(64.1)$ & $0.001^{*}$ \\
\hline Vomiting & $2(14.3)$ & $14(35.9)$ & 0.131 \\
\hline Diarrhea & $2(14.3)$ & $8(20.5)$ & 0.609 \\
\hline Blood in stool & 0 & $6(15.4)$ & \\
\hline \multicolumn{4}{|l|}{ Risk factor: } \\
\hline Hypertension & $3(21.4)$ & $19(48.7)$ & 0.755 \\
\hline Atherosclerosis & $3(21.4)$ & $18(46.2)$ & 0.105 \\
\hline Smoking & $2(14.3)$ & $12(30.8)$ & 0.230 \\
\hline Diabetes mellitus & 0 & 0 & 0 \\
\hline \multicolumn{4}{|l|}{ Angiographic findings: } \\
\hline Type I & $7(50)$ & $4(10.3)$ & 0.016 \\
\hline Type IIA & $2(14.3)$ & $25(64.1)$ & 0.014 \\
\hline Type IIB & $5(35.7)$ & $10(25.6)$ & $0.473^{*}$ \\
\hline Dissection length $[\mathrm{cm}]$ & $3.60 \pm 1.49$ & $5.09 \pm 2.13$ & $0.019^{\star}$ \\
\hline Distance from A-L [cm] & $2.09 \pm 0.77$ & $2.68 \pm 1.19$ & 0.092 \\
\hline \multicolumn{4}{|l|}{ Treatment: } \\
\hline Single stent & 0 & $34(87.2)$ & \\
\hline Overlapping stent & 0 & $5(12.8)$ & \\
\hline Diameter of stent & 0 & $7.10 \pm 0.59$ & \\
\hline Length of stent & 0 & $66.41 \pm 25.18$ & \\
\hline Fasting days & $17.07 \pm 8.51$ & $5.13 \pm 1.30$ & $0.0001^{*}$ \\
\hline \multicolumn{4}{|l|}{ Outcome: } \\
\hline Success & $11(79)$ & $38(97.4)$ & $0.021^{\star}$ \\
\hline Failure & $3(21)$ & $1(2.6)$ & $0.021^{*}$ \\
\hline Secondary treatment & $3(21)$ & $1(2.6)$ & $0.021^{*}$ \\
\hline Follow-up & $26.50 \pm 12.95$ & $31.46 \pm 13.15$ & 0.229 \\
\hline
\end{tabular}

${ }^{*} P$-value of $<0.05$, defined as statistically significant. A-L (distance from aorta to lesion entry point). 
Table III. Conservative treatment group vs. endovascular coil assisting bare stent treatment group (demographic characteristics, symptoms, angiographic findings, treatment and outcome)

\begin{tabular}{|c|c|c|c|}
\hline Variables & Conservative $(n=14)$ & Stenting + coiling $(n=9)$ & $P$-value \\
\hline Age & $52.64 \pm 5.79$ & $55.44 \pm 4.33$ & 0.228 \\
\hline \multicolumn{4}{|l|}{ Sex: } \\
\hline Male & $12(85.7)$ & $9(100)$ & \\
\hline Female & $2(14.3)$ & 0 & \\
\hline \multicolumn{4}{|l|}{ Clinical presentation: } \\
\hline Asymptomatic & $11(78.6)$ & $0(0)$ & \\
\hline Symptomatic & $3(21.4)$ & $9(100)$ & \\
\hline Duration of symptoms & $8.81 \pm 5.90$ & $3.89 \pm 1.97$ & $0.030^{*}$ \\
\hline Abdominal pain & $3(21.4)$ & $9(100)$ & \\
\hline Nausea & $2(14.3)$ & $5(33.3)$ & 0.073 \\
\hline Vomiting & $2(14.3)$ & $3(33.3)$ & 0.279 \\
\hline Diarrhea & $2(14.3)$ & $2(22.2)$ & 0.624 \\
\hline Blood in stool & 0 & $4(44.4)$ & \\
\hline \multicolumn{4}{|l|}{ Risk factor: } \\
\hline Hypertension & $3(21.4)$ & $4(44.4)$ & 0.242 \\
\hline Atherosclerosis & $3(21.4)$ & $4(44.4)$ & 0.245 \\
\hline Smoking & $2(14.3)$ & $2(22.2)$ & 0.624 \\
\hline Diabetes mellitus & 0 & 0 & 0 \\
\hline \multicolumn{4}{|l|}{ Angiographic findings } \\
\hline Type I & $7(50)$ & 0 & \\
\hline Type IIA & $2(14.3)$ & $9(100)$ & \\
\hline Type IIB & $5(35.7)$ & 0 & \\
\hline Dissection length & $3.60 \pm 1.49$ & $4.86 \pm 1.08$ & $0.040^{*}$ \\
\hline Distance from A-L [cm] & $2.09 \pm 0.77$ & $2.33 \pm 0.52$ & 0.421 \\
\hline \multicolumn{4}{|l|}{ Treatment: } \\
\hline Single stent & 0 & $7(77.8)$ & \\
\hline Overlapping stent & 0 & $2(22.2)$ & \\
\hline Diameter of stent & 0 & $6.89 \pm 0.33$ & \\
\hline Length of stent & 0 & $55.56 \pm 13.33$ & \\
\hline Fasting days & $17.07 \pm 8.51$ & $4.11 \pm 0.60$ & $0.0001^{*}$ \\
\hline \multicolumn{4}{|l|}{ Outcome: } \\
\hline Success & $11(79)$ & $9(100)$ & \\
\hline Failure & $3(21)$ & 0 & \\
\hline Secondary treatment & $3(21)$ & 0 & \\
\hline Follow-up & $26.50 \pm 12.95$ & $20.56 \pm 6.98$ & 0.222 \\
\hline
\end{tabular}

${ }^{\star} P$-value of $<0.05$, defined as statistically significant. $A-L$ (distance from aorta to lesion entry point). 
Table IV. Endovascular bare stent treatment group vs. endovascular coil assisting bare stent treatment group (demographic characteristics, symptoms, angiographic findings, treatment and outcome)

\begin{tabular}{|c|c|c|c|}
\hline Variables & Stenting $(n=39)$ & Stenting + coiling $(n=9)$ & $P$-value \\
\hline Age & $51.84 \pm 8.10$ & $55.44 \pm 4.33$ & 0.206 \\
\hline \multicolumn{4}{|l|}{ Sex: } \\
\hline Male & $31(79.5)$ & $9(100)$ & \\
\hline Female & $8(20.5)$ & 0 & \\
\hline \multicolumn{4}{|l|}{ Clinical presentation: } \\
\hline Asymptomatic & $6(15.4)$ & $0(0)$ & \\
\hline Symptomatic & $33(84.6)$ & $9(100)$ & \\
\hline Duration of symptoms & $3.97 \pm 2.59$ & $3.89 \pm 1.97$ & 0.926 \\
\hline Abdominal pain & $33(84.6$ & $9(100)$ & \\
\hline Nausea & $25(64.1)$ & $5(33.3)$ & 0.633 \\
\hline Vomiting & $14(35.9)$ & $3(33.3)$ & 0.885 \\
\hline Diarrhea & $8(20.5)$ & $2(22.2)$ & 0.909 \\
\hline Blood in stool & $6(15.4)$ & $4(44.4)$ & 0.054 \\
\hline \multicolumn{4}{|l|}{ Risk factor: } \\
\hline Hypertension & $19(48.7)$ & $4(44.4)$ & 0.817 \\
\hline Atherosclerosis & $18(46.2)$ & $4(44.4)$ & 0.993 \\
\hline Smoking & $12(30.8)$ & $2(22.2)$ & 0.611 \\
\hline Diabetes mellitus & 0 & 0 & \\
\hline \multicolumn{4}{|l|}{ Angiographic findings: } \\
\hline Type I & $4(10.3)$ & 0 & \\
\hline Type IIA & $25(64.1)$ & $9(100)$ & \\
\hline Type IIB & $10(25.6)$ & 0 & \\
\hline Dissection length & $5.09 \pm 2.13$ & $4.86 \pm 1.08$ & 0.744 \\
\hline Distance from A-L $[\mathrm{cm}]$ & $2.68 \pm 1.19$ & $2.33 \pm 0.52$ & 0.398 \\
\hline \multicolumn{4}{|l|}{ Treatment: } \\
\hline Single stent & $34(87.2)$ & $7(77.8)$ & 0.471 \\
\hline Overlapping stent & $5(12.8)$ & $2(22.2)$ & 0.471 \\
\hline Diameter of stent & $7.10 \pm 0.59$ & $6.89 \pm 0.33$ & 0.308 \\
\hline Length of stent & $66.41 \pm 25.18$ & $55.56 \pm 13.33$ & 0.218 \\
\hline Procedure duration [min] & $67.54 \pm 7.96$ & $123.33 \pm 5.05$ & $0.001^{\star}$ \\
\hline Fasting days & $5.13 \pm 1.30$ & $4.11 \pm 0.60$ & 0.095 \\
\hline \multicolumn{4}{|l|}{ Outcome: } \\
\hline Success & $38(97.4)$ & $9(100)$ & \\
\hline Failure & $1(2.6)$ & 0 & \\
\hline Secondary treatment & $1(2.6)$ & 0 & \\
\hline Follow-up & $31.46 \pm 13.15$ & $20.56 \pm 6.98$ & $0.021^{\star}$ \\
\hline
\end{tabular}

${ }^{\star} P$-value of $<0.05$, defined as statistically significant. $A-L$ (distance from aorta to lesion entry point). 
group compared to that of the conservative group; $p$-value 0.019 and 0.040 respectively (Tables II, III). The procedure duration is shorter in endovascular bare stent group compared to the endovascular coil assisting bare stent group; $p=0.001$ (Table IV). There is no significant difference in fasting days duration between bare stent and the coil assisting bare stent groups, but fasting days are much shorter in both the bare stent and the coil assisting bare stent groups compared to the conservative group, $p=0.0001$ and $p=0.0001$ respectively (Tables $I I$ and III). Treatment outcome shows a high success rate in coil assisting bare stent in which no secondary treatment was reported, success rate $100 \%(n=9)$ (Table I) whereby both conservative and bare stent alone groups reported cases of failure and re-interventions. In conservative treatment, $78.5 \%(n=11)$ were reported as successful while 4 cases were reported to have a poor response with persistent pain which was treat- ed by stenting as secondary treatment (Table II). In the bare stent alone group $97.4 \%(n=38)$ were reported as successful (Table II). In 1 case after initial treatment, the patient underwent fasting and was discharged from the hospital after 5 days. Eleven days after discharge, the patient was readmitted with recurrence of symptoms, CTA was performed and restenosis was revealed. The patient was kept under observation for 2 days in which symptoms persisted then secondary treatment was performed whereby a $7 \mathrm{~mm}$ by $21 \mathrm{~mm}$ stent was deployed successful (Photos 3 A-D). Follow-up duration was much shorter in the coil assisting bare stent group compared to the bare stent and conservative groups, $p=0.02$ (Table IV).

\section{Discussion}

The superior mesenteric artery is the $2^{\text {nd }}$ of the three main anterior visceral blood vessels of the
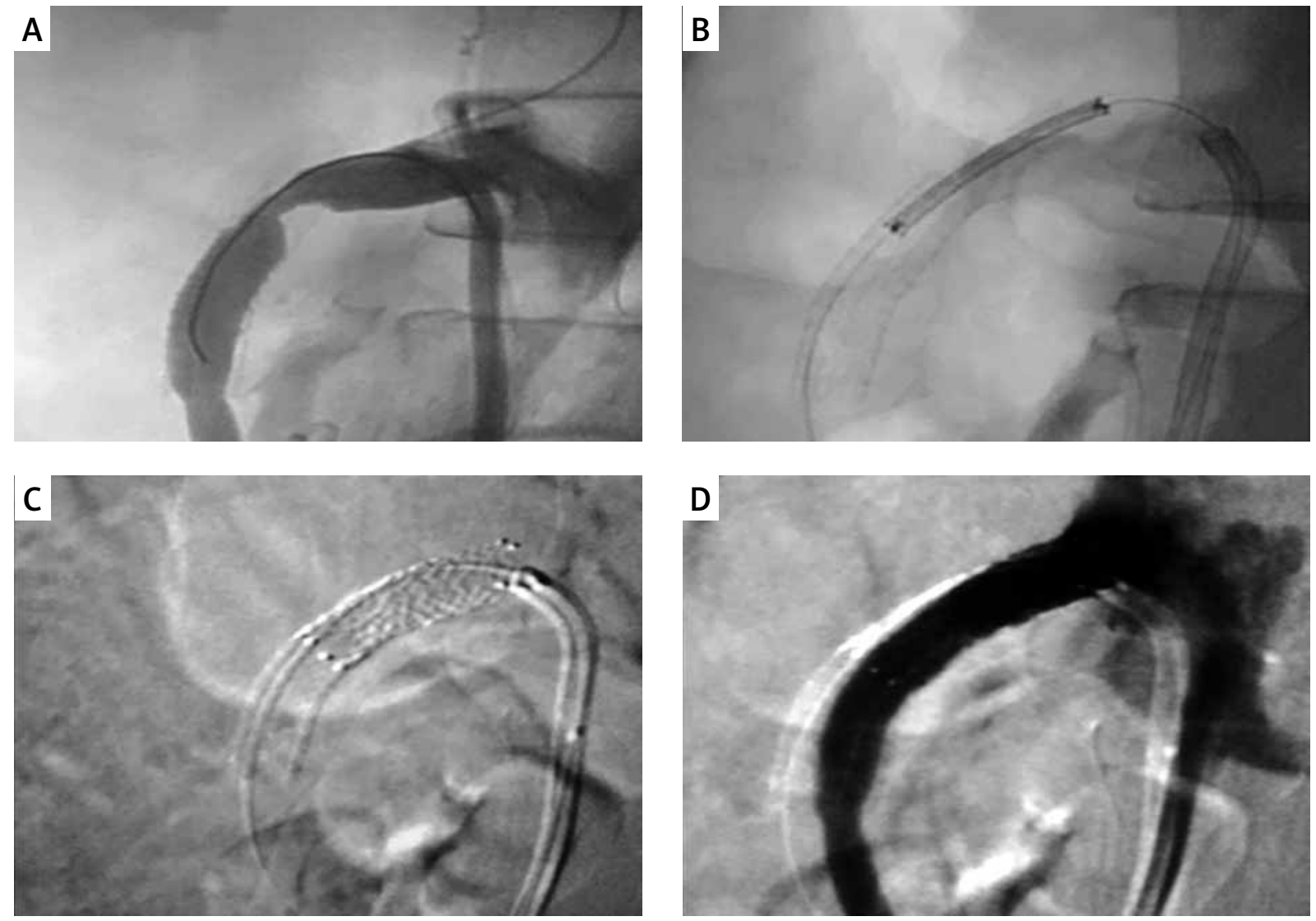

Photo 3. Management of bare stent stenosis by secondary stenting: A - angiography shows filling defect in the lumen of a bare stent at the proximal part indicating stenosis of the stent lumen, $\mathbf{B}$ - deployment of a secondary stent, $\mathbf{C}$ - secondary stent successfully deployed at the lesion site, $\mathbf{D}$ - control angiography revealing disappearance of filling defects after secondary stenting 
abdominal aorta that include the celiac and inferior mesenteric artery. Dissection of these vessels is very infrequently described and when occurring mostly the SMA is affected, with abdominal pain as the commonest presenting symptom among symptomatic patients [2, 9]. SISMAD recently has become frequently reported due to the increased use of advanced technology in diagnostic imaging studies; consequently the significant number of cases reported per year is expected to increase. With this fact in mind, there is a need to establish a universal treatment regimen based on evidence-based findings in clinical practice.

Currently, CTA is the preferred imaging modality in detecting and assessing SISMAD. Mural clot formation, intramural bleeding, intimal flap and enhanced attenuation around the SMA are significant signs of SISMAD on CTA [16]. The pathognomonic finding of SISMAD is the presence of an intimal flap in cross-sectional imaging. In general, CTA is more accurate, non-invasive and will be able to diagnose quickly especially in most cases of acute abdominal pain. On the other hand, catheter angiography is superior in assessing collateral circulation and the relationship of the lesion to branching vessels. But angiography may fail to show the lesion in case of SMA dissection in patients with a complete thrombosed false lumen (type III). Angiography is an invasive procedure; hence this procedure should be preserved and used only in those patients with worsening symptoms, who require endovascular treatment or surgical treatment. All of our final patient diagnoses were based on CTA, and the confirmatory study was done during angiographic imaging.

The treatment regimens for SISMAD patients are still debatable, but many authors recommended conservative treatment for patients with no sign of bowel ischemia while endovascular and open surgery treatment generally are reserve options for the cases where the abdominal pain has not subsided, and there is clear evidence of signs indicative of bowel ischemia or imminent rupture. In our study, we had 14 patients who received conservative treatment; 10 out of 14 who received this treatment were successful, while 4 cases were reported to have a poor response with persistent pain which was treated with stenting as a secondary treatment. Compared to endovascular treatment the number of days required for fasting was significantly higher in conservative treatment as well as the follow-up du- ration. Endovascular stent placement is considered a first-line treatment in China and in recent years is frequently used in managing SMA dissection patients. The indication for endovascular treatment is based not on the status of the symptoms or percentage of occlusion of the true lumen but rather the angiographic presentation and the presence of the collaterals circulations [3]. In our experience, we had 39 patients who received a bare stent alone and 9 patients who received a coil assisting bare stent. We selected a flexible bare self-expanding stent with a lower radial force. This type of stent is suitable for the weak vascular wall and original curved site [21, 22].

A bare stent is sufficient for opening the true lumen and allows normal flow through the SMA distal part and endothelialization of the stent with the formation of thrombus in a false lumen. In cases where the dissection lesion presents with aneurysm, coil embolization can be applied to supplement the effectiveness of the bare stent. There is no significant difference in fasting days duration between bare stent and the coil assisting bare stent groups, but fasting days are much shorter in both the bare stent and the coil assisting bare stent groups compared to the conservative group. In this case, the thrombosis of the false lumen appears to be facilitated in a short period compared to the bare stent. The procedure duration is shorter in the bare stent alone group compared to the coil assisting bare stent group while fasting days are much shorter in coil assisting bare stent compared to bare stent alone. Treatment outcome shows a high success rate in coil assisting bare stent in which no secondary treatment was reported while in bare stent 1 case developed stent restenosis and needed secondary stenting which was successful. In the case of asymptomatic patients, conservative treatment should be given priority, but all patients should be closely monitored, as it has been shown in our findings that 4 cases in this group went further to require endovascular treatment.

The study was a retrospective clinical case review in a single institute with a relatively small number of patients. Prospectively randomized clinical studies with a larger number of patients in collaboration will provide significant results.

\section{Conclusions}

Endovascular bare stent placement is a safe, effective, and successful treatment in the manage- 
ment of symptomatic SISMAD. Appropriate endovascular procedures to treat patients based on medical imaging results are a key point especially in patients with tapered vessels, longer dissection lesion, and dissection aneurysm where coil assisting bare stent shows good results. Conservative treatment should be given priority for the asymptomatic patient, but close monitoring is highly recommended.

\section{Conflict of interest}

The authors declare no conflict of interest.

\section{References}

1. Kimura Y, Kato T, Inoko M. Outcomes of treatment strategies for isolated spontaneous dissection of the superior mesenteric artery: a systematic review. Ann Vasc Surg 2018; 47: 284-90.

2. Zhao Y, Yin $\mathrm{H}$, Yao C, et al. Management of acute mesenteric ischemia: a critical review and treatment algorithm. Vasc Endovascular Surg 2016; 50: 183-92.

3. Luan JY, Li X. Computed tomography imaging features and classification of isolated dissection of the superior mesenteric artery. Eur J Vasc Endovasc Surg 2013; 46: 232-5.

4. Okada M, Ishiguchi T, Itoh H. Management of spontaneous dissection of the superior mesenteric artery. Intern Med 2004; 43 : 451-2.

5. Gobble RM, Brill ER, Rockman CB, et al. Endovascular treatment of spontaneous dissections of the superior mesenteric artery. J Vasc Surg 2009; 50: 1326-32.

6. Solis MM, Ranval TJ, McFarland DR, et al. Surgical treatment of superior mesenteric artery dissecting aneurysm and simultaneous celiac artery compression. Ann Vasc Surg 1993; 7: 457-62.

7. Sheldon PJ, Esther JB, Sheldon EL, et al. Spontaneous dissection of the superior mesenteric artery. Cardiovasc Intervent Radiol 2001; 24: 329-31.

8. Jiang G, Jia Z, Zhang X, et al. Spontaneous isolated superior mesenteric artery dissection: genetic heterogeneity of chromosome locus 5q13-14 in two male familial cases Ann Vasc Surg 2015; 29: 1019.e1-5.

9. Clair DG, Beach JM. Mesenteric ischemia. N Engl J Med 2016; 374: 959-68.

10. Ogino H. Current treatment strategy for spontaneous isolated dissection of the superior mesenteric artery. Circ J 2016; 80: 1323-5.

11. Park UJ, Kim HT, Cho WH, et al. Clinical course and angiographic changes of spontaneous isolated superior mesenteric artery dissection after conservative treatment. Surg Today 2014; 44: 2092-7.

12. Park YJ, Park KB, Kim Di, et al. A natural history of spontaneous isolated superior mesenteric artery dissection derived from follow-up after conservative treatment. J Vasc Surg 2011; 54: 1727-33.

13. Mizuno A, Iguchi H, Sawada Y, et al. Real clinical management of patients with isolated superior mesenteric artery dissection in Japan. J Cardiol 2018; 71: 155-8.
14. Kwon JH, Han YH, Lee JK. Conservative management of spontaneous isolated dissection of the superior mesenteric artery. Gastroenterol Res Pract 2017, 2017: 9623039.

15. Li N, Lu QS, Zhou J, et al. Endovascular stent placement for treatment of spontaneous isolated dissection of the superior mesenteric artery. Ann Vasc Surg 2014; 28: 445-51.

16. Kim JH, Roh BS, Lee YH, et al. Isolated spontaneous dissection of the superior mesenteric artery: percutaneous stent placement in two patients. Korean J Radiol 2004; 5: 134-8.

17. Wen D, Wang Z, Yu J, et al. Endovascular stent-graft repair of spontaneous isolated dissection of the superior mesenteric artery. Cardiovasc Intervent Radiol 2018; 41: 692-8.

18. Chang CF, Lai HC, Yao HY, et al. True lumen stenting for a spontaneously dissected superior mesenteric artery may compromise major intestinal branches and aggravate bowel ischemia. Vasc Endovasc Surg 2014; 48: 83-5.

19. Rogers C, Edelman ER. Endovascular stent design dictates experimental restenosis and thrombosis. Circulation 1995; 91: 2995-3001.

20. Jia Z, Su H, Chen W, et al. Endovascular treatment of patients with isolated mesenteric artery dissection aneurysm: bare stents alone versus stent assisted coiling Eur I Vasc Endovasc Surg 2019; 57: 400-6.

21. Furukawa H, Moriyama N. Spontaneous dissection of the superior mesenteric artery diagnosed on multidetector helical CT. J Comput Assist Tomogr 2002; 26: 143-4.

22. Zhang X, Sun Y, Chen Z, et al. Therapeutic regimen options for isolated superior mesenteric artery dissection. Vasc Endovascular Surg 2012; 46: 277-82

Received: 16.10 .2019 , accepted: 19.11.2019 